Research Article

\title{
Laparoscopic Organ-Conserving and Function-Preserving Surgical Resectional Strategy for Gastric Gastro-Intestinal Stromal Tumours (GISTs) is Safe and Effective
}

\section{Catherine Eley* and Ashraf Rasheed *}

Royal Gwent Hospital, Newport, United Kingdom

\section{ART ICLE INFO \\ Article history: \\ Received: 2 February, 2019 \\ Accepted: 21 February, 2019 \\ Published: 7 March, 2019}

\section{Keywords:}

Gastro-intestinal stromal tumour

GIST

Stomach cancer

Laparoscopy

\begin{abstract}
A B S T R A C T
Background

Gastro-intestinal stromal tumours form the most clinically important and most common group of submucosal tumours of the stomach. A gain-of-function mutation in genes coding for the KIT tyrosine kinase receptor plays a pivotal role in the differentiation and proliferation of GISTs. Surgical resection offers the only chance of cure however the consensus opinion from the European Society of Medical Oncology (ESMO) restrict laparoscopic approaches to tumours $<2 \mathrm{~cm}$ in certain anatomical locations provided surgical oncological principles are observed. This study will assess the safety, effectiveness, and functional outcomes of an anatomically-based strategy for the laparoscopic resection of gastric GISTs.

Methods

All symptomatic gastric GISTs diagnosed during a 5 years period were considered for minimal access surgical resection. Lesions were removed according to a structured anatomical approach. The strategy was designed to conserve the organ and preserve sphincter (lower oesophageal and pyloric) function while adhering to oncological principles. A prospective database included patients' demographics, clinical features, imaging, procedure type, operative findings, complications, histopathology, immunohistochemistry, mutation status and risk stratification. Clinical and radiological follow-up was recorded.

Results

Thirty-two (32) cases of suspected gastric GISTs were considered for minimal access resection during the study. Twenty-three patients (72\%) underwent a laparoscopic wedge resection, six patients (19\%) underwent an extra-gastric anterior resection and three patients $(9 \%)$ underwent trans-gastric posterior resection. 97\% were histologically confirmed GIST, all achieving R0 excision. 97\% were disease free at 20 months.

\section{Conclusion}

The authors have demonstrated that an anatomically-based surgical strategy for the laparoscopic resection of gastric submucosal tumours is safe and enjoys an oncological and functional success. This, along with the recognised reduced morbidity associated with minimal access surgery lend support to the notion that laparoscopic surgery might be the preferable approach for small to medium sized gastric GISTs.
\end{abstract}

(C) 2019 Catherine Eley. Hosting by Science Repository. 


\section{Introduction}

Gastro-intestinal stromal tumours (GISTs) form the most clinically important and most common group of submucosal tumours of the stomach albeit accounting for $<1 \%$ of all gastrointestinal (GI) malignancies. Annual incidence is estimated to be in the region of 10 per million persons and can occur anywhere along the GI tract [1, 2]. GISTs most frequently occur in the stomach $(60 \%)$, followed by small bowel (30\%), duodenum (5\%), rectum (3-4\%) and colon (1-2\%); and demonstrate local, intra-abdominal and metastatic spread, almost never involving lymphatics [2,3].

Until recently, GISTs have been misclassified as 'true' smooth muscle tumours (leiomyomas, leiomyoblastomas, leiomyosarcomas) but can now be readily distinguished from myogenic, neurogenic or tumours of other mesenchymal origin [4]. Their considerable variability in cellular differentiation led to the recognition of the interstitial pacemaker cells of Cajal (ICC), or more possibly their precursors, as the origin of GISTs [5]. These form the interface between the autonomic innervation of smooth muscle and the gastro-intestinal wall and are found within the myenteric plexus, submucosa and muscularis propria of the GI tract [6, 7].

Most GISTs are characterised by the expression of activated KIT receptor tyrosine kinase (CD 117 antigen) [1-12]. A gain-of-function mutation in genes coding for the KIT tyrosine kinase receptor is considered to play a pivotal role in the differentiation and proliferation of this tumour [6, 8]. In addition, a smaller proportion of GISTs (5-10\%) have mutations in the gene encoding platelet-derived growth factor $\alpha$ (PDGFR $\alpha$ ) that is a tyrosine kinase receptor homologous to KIT [9]. Furthermore, there is still about $10-15 \%$ of GISTs that lack any mutations in the above genes, and these are known as wild-type GIST [4]. These differing characteristics are increasingly proving to be associated with specific phenotypes that are ultimately becoming more important clinically with the advent of targeted therapy. Excellent results in patients with unresectable or metastatic GIST have been shown with imatinib mesylate, a tyrosine kinase receptor antagonist [1, 10, 12]. Complete surgical resection however remains to be only modality that offers a chance for a cure and is the treatment of choice for primary localised GISTs [11].Traditionally, resection has been achieved by open surgery with no clear guidance or recommendations of when and how to apply oncological minimal access techniques in GISTs. The European Society for Medical Oncology (ESMO) advocates a laparoscopic approach for small $<2 \mathrm{~cm}$ sized tumours; with the National Comprehensive Cancer Network (NCCN) expanding the previously set boundaries of laparoscopic approaches based on a series of larger GISTs tumours that are resected laparoscopically observing the surgical oncological principles at all times $[1,2]$

\section{Objectives}

To assess the safety, effectiveness, and functional outcomes of an anatomically-based surgical strategy for the laparoscopic resection of gastric GISTs.

\section{Methods}

All symptomatic gastric GISTs that were diagnosed during a 5 years period were considered for minimal access surgical resection. Lesions were removed according to a structured anatomical approach. Sound oncological principles were adhered to by aiming for tumour-free resection margins (R0), avoiding tumour handling or rupture. The strategy was designed to conserve the organ (stomach) and preserve sphincter (lower oesophageal and pyloric) function. Systematic lymphadenectomy was not performed as judged to be unnecessary. A prospective database was then constructed to include patients' demographics, clinical features, imaging, procedure type, operative findings, complications, histopathology, immunohistochemistry, mutation status and risk stratification. Clinical and radiological followup was recorded.

\section{Results}

Thirty-two (32) cases of suspected gastric GISTs that were considered for minimal access resection during the study were entered into the database. Seventeen (53\%) were males and fifteen (47\%) females with a mean age of 66 years (range 39-83 years). Symptoms included haematemesis, abdominal pain or malaena. All patients underwent at least a pre-operative contrast enhanced computed tomography (CT) and oesophago-gastro-duodenoscopy (OGD) to localise and characterise the lesion. Further investigations were performed only if deemed necessary. Twenty-three patients (72\%) underwent a laparoscopic wedge resection, six patients (19\%) underwent an extra-gastric anterior resection and three patients $(9 \%)$ underwent a trans-gastric posterior resection. There were no conversions to open, no major intra-operative complications and no episodes of tumour rupture. There were no major immediate or early surgical complications.

Thirty-one (31) cases (97\%) were proven to be GIST while 1 case (3\%) was histologically a benign leiomyoma. Mean lesion size was $44 \mathrm{~mm}$ (range $20-90 \mathrm{~mm}$ ). Complete resection (R0) was achieved in $100 \%$ of cases with a mean postoperative length of stay of 5.6 days.

Morbidity was minimal with 1 staple line leak that required a reoperation and 1 splenic capsule haematoma that was managed conservatively. There was no peri-operative (30 day or in hospital) mortality.

There is no good quality evidence to inform us of the optimal modality or frequency of post-operative image surveillance for these patients [1, 2]; in our institute, low risk GISTs are followed up with yearly abdominal and pelvic contrast-enhanced AP-CT (CE-CT) scan for at least 10 years, but high risk GISTs are surveilled more intensely to pick up metastasis or recurrences at an early stage i.e. with the lowest tumour load and offer GISTectomy for singular recurrence plus/minus adjuvant therapy; and adjuvant therapy for GISTosis (multiple GIST recurrence). For this high-risk group, we recommend 4-monthly AP CE-CTs for the first year and 6 monthly the year after and then yearly up to 10 years thereafter. The median follow-up in our cohort was 20 months with no reported dysphagia, reflux or dumping syndrome and 30 patients with confirmed GIST (97\%) had negative follow-up CT scans i.e. no recurrence or metastasis.

\section{Overall Survival}

One patient with a high-risk tumour 'size and mitotic index' succumbed to progressive disease at the $11^{\text {th }}$ post-operative month. 


\section{Discussion}

The management of GISTs continues to evolve rapidly. Several authors have now demonstrated the effectiveness of the laparoscopic management of gastric GISTs and this approach supported further by a meta-analysis comparing this to open approach [13]. It is important not to forget however that the concept of the laparoscopic resection of gastric tumours is only two decades old and that the understanding of GISTs and their malignant potential is still in its infancy. Nonetheless, expansion of minimal access approaches to surgical oncology lend itself nicely to management of gastric GISTs.

Surgery remains the mainstay of curative therapy for non-metastatic lesions. What is not established however, is the preferred operative approach and extent of surgical resection. The current consensus varies between Europe and USA with ESMO advocating laparoscopic surgery for small GISTs $(<2 \mathrm{~cm})$ due to the possibility of breaching oncological principles upon handling of larger GISTs [2]; and NCCN describing a laparoscopic approach to tumours up to and greater than $5 \mathrm{~cm}$ [1]. Larger GIST tumours i.e. $>10 \mathrm{~cm}$ require a sizable incision for non-traumatic retrieval from the abdomen and in these cases, it might be easier, quicker and safer to perform an open operation.

One should remember that the aim of surgery for GIST is complete, nontraumatic (without breach of the pseudo-capsule) removal of the tumour achieving negative resection margins without the need for lymphadenectomy. And if the aforementioned can be accomplished laparoscopically with preservation of stomach's function and maintaining the integrity of the gastric sphincters then this would be beneficial.

Difficulties may arise in the laparoscopic approaches due to the tumour locations making certain resections more technically challenging. This is mainly due to factors such as tumour size, access to the tumour and gastric mobility. Our adopted strategy attempted to observe the oncological surgical principles with conservation of the organ and preservation of the function. This requires accurate pre-operative mapping with at least OGD and CE-CT. Other imaging modalities such as an Endoscopic Ultrasound (EUS) or Magnetic Resonance Imaging (MRI) with or without Positron Emission Tomography (PET) might also be needed. Distance from the gastro-oesophageal junction (GOJ) must be measured and exophytic/endophytic characteristics of the tumour should be noted. It is only with a thorough pre-operative planning that the most appropriate approach can be chosen, and the best chance of cure is achieved.

We recognise that the proximity of the lesion to either of the sphincters to be an important factor in the choice of the type of resection. We decided that $3 \mathrm{~cm}$ distance of the lesion from either sphincter to be the minimum distance to allow an oncologically sound resection and conservation and preservation of sphincter function. We called this $3 \mathrm{~cm}$ distance from the sphincter as the sphincter zone. We chose $3 \mathrm{~cm}$ as the distance based on the fact that $1 \mathrm{~cm}$ will be consumed by the staple line leaving us with $2 \mathrm{~cm}$ to cater for R0 negative margins and sphincter preservation. If the GIST lies within this $3 \mathrm{~cm}$ sphincter zone, we don't attempt to preserve the sphincter so not to breach the oncological principles or risk disrupting local anatomy, compromising the sphincter and leading to a poor functional outcome. And based on the above, lesions located within the Gastro-Oesophageal sphincter zone would require proximal gastric resection with an addition of anti-reflux procedure to prevent reflux (Figure 1) while a distally located gastric GIST that lies within the pyloric sphincter zone might need a distal gastrectomy.
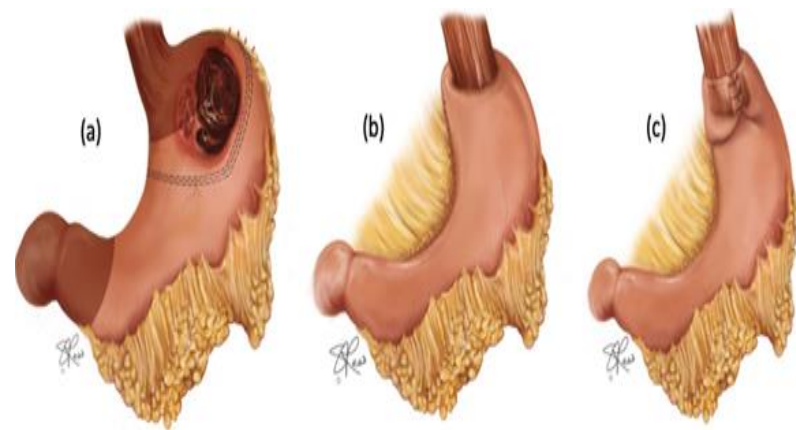

Figure 1: (a) Tumour within the $3 \mathrm{~cm}$ sphincter zone proximally (b) requiring proximal gastrectomy (c)with anti-reflux procedure

When the lesion is located outside of the sphincter zone, a structured laparoscopic approach to these lesions can avoid unnecessary resection whilst still achieving a sound oncological resection and favourable functional outcome. Lymphadenectomy is unnecessary given the rarity of lymph node spread. So for example, a lesser curve lesions outside the sphincter zone can be treated by a wedge resection with an adequate margin of $1 \mathrm{~cm}$. (Figure 2).

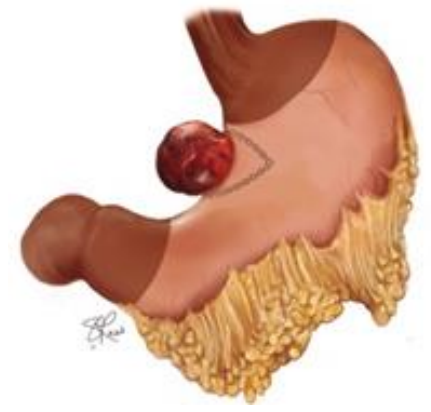

Figure 2: Staple line depicting wedge resection of lesser curve GIST.

Greater curve lesions can be resected adopting an extra-gastric tangential resection. This approach can also be used to tackle lesions on the anterior wall of the stomach with or without endoscopic guidance (Figure 3).
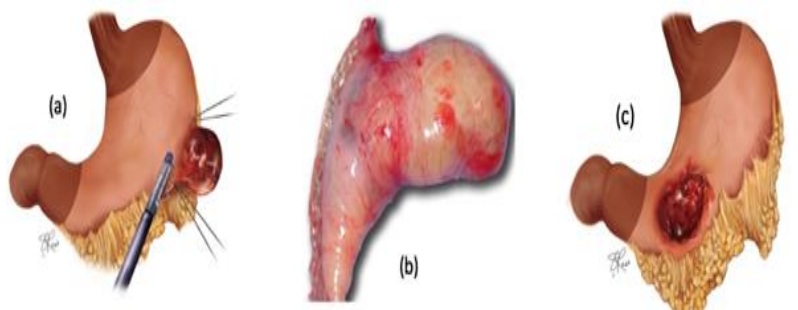

Figure 3: (a) Illustration depicting the approach to a GIST on the greater curve of the stomach (b) with surgical specimen following extra-gastric tangential resection, (c) anterior gastric wall GIST, not encroaching on the sphincter zones

Posterior wall endophytic GISTs can be approached trans-gastrically via an anterior gastrotomy, followed by tangential resection; with or without guidance of intra-operative endoscopy. Whereas exophytic GISTs on the 
posterior wall of the stomach can be approached by dividing the gastrocolic ligament and performing an extra-gastric tangential resection with aid of intra-operative endoscopy; ensuring complete excision with adequacy of margins (Figure 4).
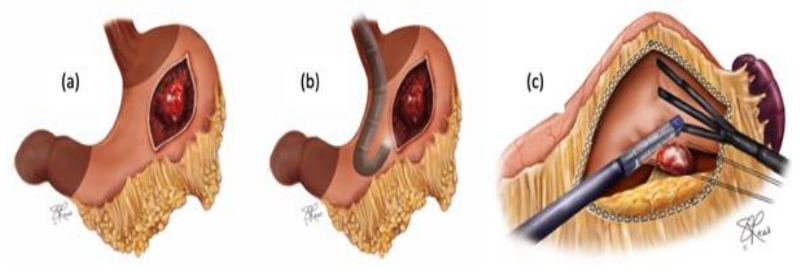

Figure 4: (a) Anterior gastrotomy approach to posterior wall endophytic GIST in order to perform trans-gastric tangential resection (b) with endoscopic guidance (c) Access to posterior stomach wall GIST for tangential resection of exophytic GIST

The unpredictable behaviour of GISTs makes long term follow up a necessity. Most recurrences tend to occur within the first two years post resection with rate of recurrence based on risk stratification especially with factors such as size and mitotic index [1]. Metastases however can present up to 10-15 years post primary GIST resection [14]. GIST should always be managed within a multidisciplinary team (MDT) setting in a specialist cancer centre. Survival rates for inoperable and/or metastatic GIST have improved with the advent of tyrosine kinase inhibitors, however secondary resistance to imatinib is now being realised and chemotherapy and radiotherapy currently have a limited or no role in the management of GIST [1,2].

The role of surgery in advanced metastatic GIST remains unclear. It can be used to aid with palliation for bleeding or obstructing lesions but does not seem to benefit progressive disease. Those with stable disease or disease with limited progression on kinase inhibitor therapy show increased survival after debulking procedures.

\section{Conclusions}

This cohort confirms that a structured anatomically-based surgical strategy to aid minimal access resection of gastric submucosal tumours is feasible, safe and enjoys an oncological and functional success. This, along with the recognised reduced morbidity associated with minimal access surgery lend support to the notion that laparoscopic surgery is the preferable approach for small to medium sized gastric GISTs.

\section{Competing Interests}

There are no competing interests to declare.

\section{REFERENCES}

1. 11. Demetri GD, von Mehren M, Antonescu CR, DeMatteo RP, Ganjoo KN, et al. (2010) NCCN Task Force report: update on the management of patients with gastrointestinal stromal tumors. J Natl Compr Canc Netw 8: 1-41. [Crossref]

2. Casali PG, Abecassis N, Bauer S, Biagini R, Bialek S, et al. (2018) on behalf of the ESMO Guidelines Committee and EURACAN. Gastrointestinal stromal tumours: ESMO-EURACAN Clinical Practice Guidelines for diagnosis, treatment and follow up. Ann Oncol 29: 6878. [Crossref]

3. Jamkowski J, Sampliner R, Kerr D, Fong Yuman (2008) Gastrointestinal Oncology; A Critial Multidisciplinary team approach.Blackwell Publishing p194-228.

4. Fletcher CD, Berman JJ, Corless C, Gorstein F, Lasota J, et al. (2002) Diagnosis of gastrointestinal stromal tumors: A consensus approach. Hum Pathol 33: 459-465. [Crossref]

5. Graadt van Roggen JF, van Velthuysen ML, Hogendoorn PC (2001) The histopathological differential diagnosis of gastrointestinal stromal tumours. J Clin Pathol 54: 96-102. [Crossref]

6. Miettinen M, Lasota J (2006) Gastrointestinal stromal tumors: pathology and prognosis at different sites. Semin Diagn Pathol 23: 7083. [Crossref]

7. DeMatteo RP, Lewis JJ, Leung D, Mudan SS, Woodruff JM, et al. (2000) Two hundred gastrointestinal stromal tumors: recurrence patterns and prognostic factors for survival. Ann Surg 231: 51-58. [Crossref]

8. Corless CL, Fletcher JA, Heinrich MC (2004) Biology of Gastrointestinal Stromal Tumors. J Clin Oncol 22: 3813-3825. [Crossref]

9. Corless CL, Schroeder A, Griffith D, Town A, McGreevey L, et al. (2005) PDGFRA mutations in gastrointestinal stromal tumors: frequency, spectrum and in vitro sensitivity to imatinib. J Clin Oncol 23: 5357-5364. [Crossref]

10. Kitamura Y, Hirota S, Nishida T (2003) Gastrointestinal stromal tumors (GIST): a model for molecule-based diagnosis and treatment of solid tumours. Cancer Sci 94: 315-320. [Crossref]

11. Chaudhry UI, DeMatteo RP (2009) Management of resectable gastrointestinal stromal tumor. Hematol Oncol Clin North Am 23: 7996. [Crossref]

12. Shi YN, Li Y, Wang LP, Wang ZH, Liang XB, et al. (2017) Gastrointestinal stromal tumor (GIST) with liver metastases; An 18year experience from the GIST cooperation group in North China. Medicine (Baltimore) 96: e8240. [Crossref]

13. Ohtani H, Maeda K, Noda E, Nagahara H, Shibutani M, et al. (2013) Meta-analysis of laparoscopic and open surgery for gastric gastrointestinal stromal tumor. Anticancer Res 33: 5031-5041. [Crossref]

14. Miettinen M, Sobin L, Hm Lasota J (2005) Gastrointestinal stromal tumors of the stomach: a clinicopathologic, immunohistochemical, and molecular genetic study of 1765 cases with long-term follow-up. Am J Surg Pathol 29: 52-68. [Crossref] 